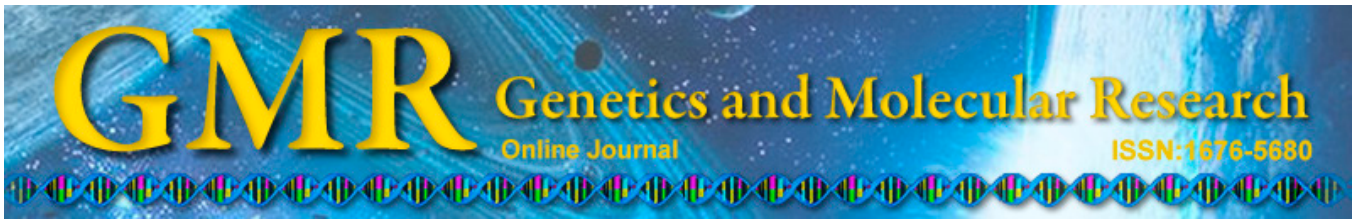

\title{
Bone morphogenetic protein 2 and decorin expression in old fracture fragments and surrounding tissues
}

\author{
X.G. Han ${ }^{1}$, D.K. Wang ${ }^{2}$, F. Gao ${ }^{3}$, R.H. Liu ${ }^{1}$ and Z.G. Bi ${ }^{1}$ \\ ${ }^{1}$ Department of Orthopedics, \\ First Affiliated Hospital of Harbin Medical University, Harbin, \\ Heilongjiang Province, China \\ ${ }^{2}$ Department of Radiology, \\ First Affiliated Hospital of Harbin Medical University, Harbin, \\ Heilongjiang Province, China \\ ${ }^{3}$ The Veins Allocation Center of Clinic Service, \\ First Affiliated Hospital of Harbin Medical University, Harbin, \\ Heilongjiang Province, China \\ Corresponding author: Z.G. Bi \\ E-mail: buhgwin@163.com
}

Genet. Mol. Res. 14 (3): 11063-11072 (2015)

Received November 12, 2014

Accepted May 8, 2015

Published September 21, 2015

DOI http://dx.doi.org/10.4238/2015.September.21.19

\begin{abstract}
Bone morphogenetic protein 2 (BMP-2) can promote fracture healing. Although the complex role BMP-2 in bone formation is increasingly understood, the role of endogenous BMP-2 in nonunion remains unclear. Decorin $(\mathrm{DCN})$ can promote the formation of bone matrix and calcium deposition to control bone morphogenesis. In this study, tissue composition and expression of BMP-2 and DCN were detected in different parts of old fracture zones to explore inherent anti-fibrotic ability and osteogenesis. Twenty-three patients were selected, including eight cases of delayed union and 15 cases of nonunion. Average duration of delayed union or nonunion was 15 months. Fracture fragments and surrounding tissues, including bone
\end{abstract}


grafts, marrow cavity contents, and sticking scars, were categorically sampled during surgery. Through observation and histological testing, component comparisons were made between fracture fragments and surrounding tissue. The expression levels of DCN and BMP-2 in different tissues were detected by immunohistochemical staining and real-time polymerase chain reaction. The expression of DCN and BMP2 in different parts of the nonunion area showed that, compared with bone graft and marrow cavity contents, sticking scars had the highest expression of BMP-2. Compared with the marrow cavity contents and sticking scars, bone grafts had the highest expression of DCN. The low antifibrotic and osteogenic activity of the nonunion area was associated with non-co-expression of BMP-2 and DCN. Therefore, the co-injection of osteogenic factor BMP and DCN into the nonunion area can improve the induction of bone formation and enhance the conversion of the old scar, thereby achieving better nonunion treatment.

Key words: Fracture nonunion; Bone morphogenetic protein 2; BMP-2; Decorin; Osteogenesis transformation

\section{INTRODUCTION}

There has been significant progress in the understanding of the molecular and cellular mechanisms of bone healing in fracture treatment in recent years. However, 5-10\% of clinical fractures exhibit delayed union or nonunion (Praemer et al., 1992). Normal fracture healing requires the interaction of different cell types, and bone morphogenetic proteins (BMPs), including BMP-2, BMP-4, and BMP-7, play a central role in the formation of bone tissue (Groeneveld and Burger, 2000; Reddi, 2001). Several studies have confirmed the promotion of BMPs in fracture healing in animal models, indicating that exogenous BMPs can promote healing in nonunion tissue (Johnson et al., 1988a,b, 1990, 1992). At present, there is little understanding of the cellular microenvironment of the human bone nonunion region and the pathological process leading to the formation of fibrous scars (Schmitz et al., 1990; Hietaniemi et al., 1996, 1998). It has been reported that the genetic transformation from osteoblasts to fibroblasts is caused by an absence of signals resulting from osteoblast differentiation factors (such as BMPs) in space and time. According to the theory that good complex signals result in distortion, it can be concluded that the application of exogenous BMP can promote osteoblast differentiation (Guerkov et al., 2001). However, there has been no report on this distortion applied to fracture nonunion, and the levels of osteogenic BMPs in fracture nonunion is unclear.

Decorin (DCN) is a small leucine-rich proteoglycan with a molecular weight of 90 $140 \mathrm{kDa}$. It can regulate cell growth, growth factor activity, and the accumulation of the extracellular matrix. DCN is concentrated in the active areas of bone calcium, and is expressed in bone tissues during the development of mature bone and most collagen-enriching mesenchymal cells. The roles of DCN include adjusting transforming growth factor activity and the generation of collagen microfibrils. In addition, DCN plays an important role in regulating mineralization by directly binding to the lateral diffusion osteoid, which is limited to the new fetal bone matrix. This bone matrix exists in all stages of bone formation and in adult bones. $\mathrm{DCN}$ is also involved in ossification and binding to the specific surfaces of hydroxyapatite 
crystals, and is therefore able to control crystal morphogenesis. Moreover, decorin regulates hydroxyapatite generation in the gelatin system; it has a high affinity for hydroxyapatite and affects nucleation, location, size, and calcium phosphate formation.

In this study, tissue composition and the expression levels of BMP-2 and DCN were determined in different parts of the nonunion region to investigate inherent anti-fibrotic ability and osteogenesis. We explored the potential of a combination of BMP-2 and DCN for improving the treatment of bone nonunion.

\section{MATERIAL AND METHODS}

\section{Design}

The tissue compositions and expression levels of BMP-2 and DCN were detected in different parts of the nonunion region.

\section{Time and place}

The study was conducted between 2009 and 2010 at the Center Laboratory of the First Affiliated Hospital of Harbin Medical University.

\section{Reagents and materials}

The TRIzol kit was from Invitrogen (Shanghai, China); oligo-(dT) 15 was from TaKaRa Co., Ltd. (Japan) (D510); the ribonuclease inhibitor was from TaKaRa Co., Ltd. (Japan) (D2310A), the M-MLV reverse transcriptase was from Promega (Chicago, IL, USA) (M170A), and the DNA Marker I was from Tiangen Biotech (MD101) (Beijing, China). The SYBR Green polymerase chain reaction (PCR) Master Mix kits were purchased from American Applied Biosystems Company, Lot No. 0612286, and the PRISM 7700 fluorescent quantitative PCR instrument was from Applied Biosystems, USA. BMP and DCN monoclonal antibodies were from the Wuhan Boster Company (Wuhan, China). The PowerVision ${ }^{\mathrm{TM}}$ immunohistochemical reagents were from Beijing Zhongshan Golden Bridge Biotechnology Co., Ltd. (Beijing, China). Clean benches were from the Lixin Company (Beijing, China); a TGL-1G high-speed desktop centrifuge was from the Shanghai Analytical Instrument Factory (Shanghai, China); and the $-80^{\circ} \mathrm{C}$ ultra-low temperature freezer was from the SANYO Company (Japan).

\section{Tissue collection}

Eleven limb bone nonunion patients (mean age of 40 years, range 27-81 years) were selected. Nonunion was defined as failure of the fracture to heal 6 months or more after surgery or non-surgical treatment. The average time from fracture to sample collection was 11 months (range 6-30 months). All patients with a chief complaint of limb pain, shortening, and dysfunction were diagnosed by imaging, physical examination, laboratory tests, and intraoperative findings. We collected the fracture and scar tissue during surgery, which was divided into bone stump tissue, marrow cavity contents, and sticking bone scars according to the sites; the samples were stored at $-80^{\circ} \mathrm{C}$ in a refrigerator. 


\section{Tissue fixation and embedding}

The tissues obtained were fixed for $48 \mathrm{~h}$ in $10 \%$ neutral formalin. Samples were dehydrated by concentration-gradient ethanol, embedded in paraffin, and sliced into 5-6- $\mu \mathrm{m}$ slices for immunohistochemical analysis. Bone tissues were demineralized before being embedded and sliced. All slices were encoded to prevent deviation in the immunohistochemical score, and hematoxylin and eosin staining was used for histological analysis and comparison.

\section{Immunohistochemical analysis}

The tissue sections were baked at $60^{\circ} \mathrm{C}$ in an incubator for 20 min, dewaxed in xylene, and dehydrated in anhydrous ethanol. After addition of ethylene diaminetetraacetic acid in boiling water, $\mathrm{pH} \mathrm{8.0,} \mathrm{the} \mathrm{samples} \mathrm{were} \mathrm{heated} \mathrm{for} 10 \mathrm{~min}$ in the autoclave. After cooling to room temperature, dewaxing, hydration, and antigen retrieval, anti-rabbit antibody BMP-2 (1:50) was added drop-wise and left to stand overnight at $4^{\circ} \mathrm{C}$. Secondary antibody (40-50 $\mu \mathrm{L}$ ) was then added and allowed to stand at room temperature. After 3,3'-diaminobenzidine coloration for 5-10 min, a microscope was used to assess the degree of staining; after washing for $10 \mathrm{~min}$, samples were counterstained with hematoxylin and eosin for $2 \mathrm{~min}$ and rinsed again for $10 \mathrm{~min}$. Dehydration, transparent section sealing, and microscopic examination were performed in order.

\section{Quantification}

Five immunohistochemical sections that did not overlap were randomly selected under a 400X magnification light microscope to avoid sampling error. Morphological parameters and optical density of the tissues in the sections were measured using a Motic digital medical image analysis system (Motic Med 6.0). The nonspecific gray background staining was automatically subtracted and the extent of positive staining was calculated to show the staining intensity and content; the average percentage of staining was calculated in all five regions.

\section{Real-time PCR}

After grinding in liquid nitrogen, total RNA was extracted by the conventional TRIzol method, and detected by formaldehyde denaturing on agarose gel. In accordance with the SYBR Green PCR Master Mix kit manufacturer protocol, the RNA was reverse transcribed into cDNA and real-time PCRs were performed on the ABI PRISM 7700 fluorescence quantitative PCR instrument. The reaction system comprised a total of $20 \mu \mathrm{L}$ PCR system containing $2 \mathrm{X} 10 \mu \mathrm{L}$ SYBR Green PCR Master Mix; $2 \mu \mathrm{L}$ cDNA; $1 \mu \mathrm{L}$ upstream primer; and $1 \mu \mathrm{L}$ downstream primer. The product lengths and primer sequences are shown in Table 1. The amplification conditions were: denaturation at $95^{\circ} \mathrm{C}$ for $10 \mathrm{~min}, 95^{\circ} \mathrm{C}$ for $15 \mathrm{~s}, 60^{\circ} \mathrm{C}$ for $30 \mathrm{~s}$, $72^{\circ} \mathrm{C}$ for $45 \mathrm{~s}$, for a total of 40 cycles, and final extension at $72^{\circ} \mathrm{C}$ for 3 min. Taking the $\beta$-actin gene as an endogenous reference gene, the melting curve of the amplified product was drawn after the reaction. The expression specificity of the PCR products was monitored by analyzing the melting curve; according to the real-time quantitative $2^{-\Delta \Delta \mathrm{Ct}}$ method, the gene expression differences between samples were compared. 
Table 1. Primer sequences and predicted product sizes.

\begin{tabular}{|c|c|c|}
\hline & Primer sequence & Predicted size (bp) \\
\hline BMP-2 & $\begin{array}{l}\text { F: 5'-GGGAGCCACAATCCAGTCA-3' } \\
\text { R: 5'-GGGAGCCACAATCCAGTCA-3' }\end{array}$ & 261 \\
\hline Decorin & $\begin{array}{l}\text { F: 5'-TAGAACTGGGCACCAATCCG-3' } \\
\text { R: } 5^{\prime} \text {-CGTAAGGGAAGGAGGAAGAC-3' }\end{array}$ & 137 \\
\hline$\beta$-actin & $\begin{array}{l}\text { F: 5'-CGCACCACTGGCATTGTCAT-3' } \\
\text { R: 5'-TTCTCCTTGATGTCACGCAC-3' }\end{array}$ & 200 \\
\hline
\end{tabular}

\section{Statistical analysis}

The experimental results are reported as means \pm standard deviation; statistical analysis among groups was performed using one-way analysis of variance with the SPSS 10.0 software; $\mathrm{P}<0.05$ was considered to be statistically significant.

\section{RESULTS}

\section{Histological examination}

By general observation, the canal was closed in the nonunion region, bone sclerosis was observed in the fracture fragments, fiber connection was mainly observed in the fracture fragments, and the surrounding tissues were all tough scar tissues. Under the microscope (Figure 1), delayed union and nonunion areas comprised a mix of different types of tissues: fracture fragments and surrounding tissues were mainly subject to fibrosis, in which the formation of new blood vessels could be seen, and a small amount of woven bone could be seen nearby. In these woven bones, Gergen Bauer's cells grew along the osteoid as cubes, suggesting active bone formations. A large number of cartilage cells existed in the intramedullary tissues, and there was no new bone and neovascularization. Bone marrow occlusion was observed, and in the fibrous tissue of adjacent bone and the gap of bone fractures, there were internal cartilage ossifications and fibrous ossifications. Scattered lamellar bone fragments were observed in some samples; these fractures were surrounded by osteoclasts, and there was a lack of osteoblasts.
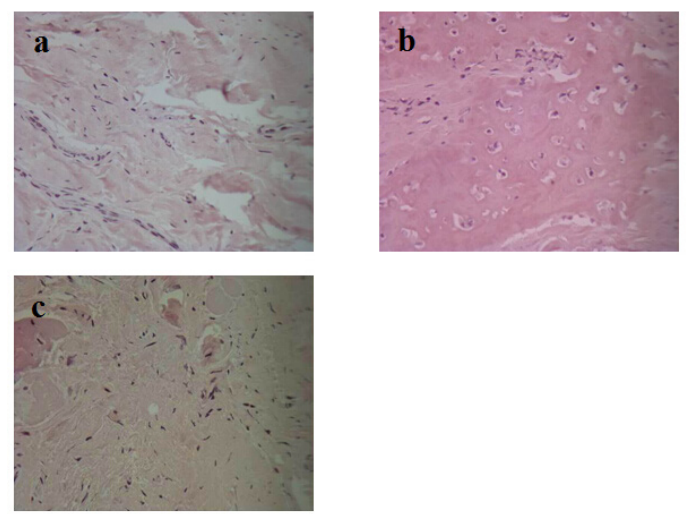

Figure 1. Histological examination (400X magnification microscopy). a. Fracture fragments; b. medullary cavity contents; c. sticking scar. Fracture fragments and their surrounding sticking scars were mainly fibrotic tissues, in which neovascularization and a small amount of woven bone can be seen. A large number of cartilage cells can be seen in the intramedullary tissues; there were no new bone and neovascular tissues. 


\section{Immunohistochemistry observation}

In general, the depth of BMP-2 staining in the cytoplasm increased with increasing proximity to the new bone formation region, and there was some staining of the Golgi apparatus, showing that BMP-2 was locally generated. A wide variety of cells, including epithelial cells, smooth muscle cells around the small blood vessels, fusiform fibroblast-like cells, and chondrocyte cells, showed positive staining in the fibrous tissues, indicating osteogenesis. There was no difference in the immunostaining of fibrous tissue between the samples with and without new bone. There was no positive BMP staining in the extracellular matrix or the fibrous tissue space. Sub-parts of view, fracture fragments were mainly fibrotic tissues and BMP-2 staining was negative. In the surrounding tissues, especially in the sticking scars and posted plate scars, neovascular and woven bone filled in a lot of the fibrous tissues, and in the vicinity there were stained cells, indicating BMP-2 expression. There was a small amount of cartilage with positive staining in the cytoplasm, without expression in fibrous tissues of the closed medullary cavity (Figure 2). DCN expression was extensive in the interstitial fracture fragments. There was no positive staining of cartilage cells in the medullary cavity. DCN expression in the sticking scars was close to perivascular.
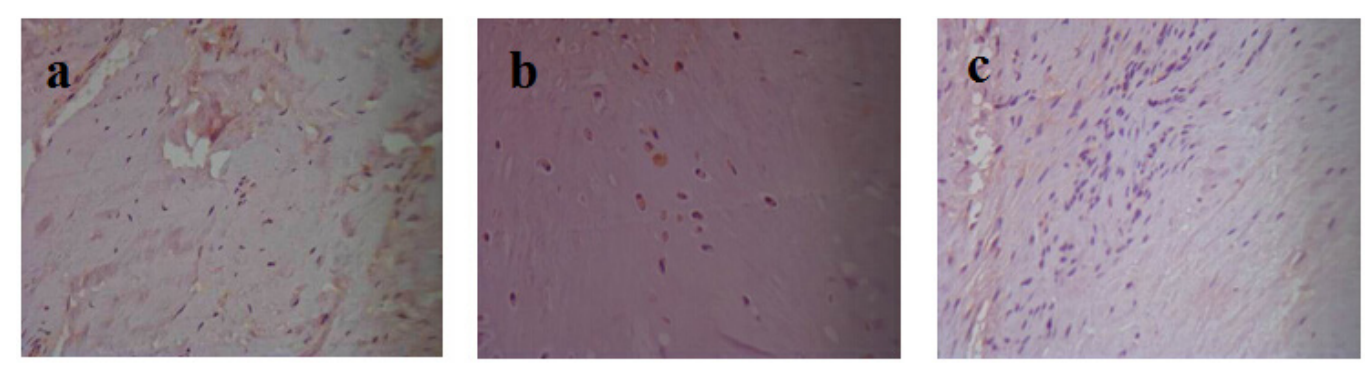

Figure 2. Immunohistochemistry: bone morphogenetic protein 2 (BMP-2) expression (400X magnification microscopy). a. Fracture fragments; b. medullary cavity contents; c. sticking scar. Fracture fragments were mainly fibrotic tissues and BMP-2 staining was negative. In the surrounding tissues, especially in the sticking scars and posted plate scars, neovascular and woven bone filled in much of the fibrous tissues, and there were stained cells indicating BMP-2 expression in the vicinity. There were a small amount of positively stained cartilage in the cytoplasm, but no expression in the fibrous tissues within the closed medullary cavity.

Through image analysis software, we randomly selected five visible stained cell regions and determined the positive expression rate. The SPSS 10.0 software was used for statistical analysis. The rate of expression of BMP-2 was highest in the posted bone scar group, and was low in the bone ends and canal content group; there was a significant difference between the two groups $(\mathrm{P}<0.05)$. There was no significant difference between the other two groups. The fracture fragment group had the highest DCN expression, with significant differences from the other two groups; the least significant difference analysis showed that between the fracture fragment group and the other two groups, $\mathrm{P}<0.05$; between the other two groups, $\mathrm{P}>0.05$ (Table 2).

\section{Real-time PCR}

Real-time quantitative calculation was based on the $2^{-\Delta \Delta C t}$ method. The expression levels of BMP-2 and DCN in soft tissue around the bone ends were set to 1 . The expression 
levels in the bone ends group, the marrow cavity contents group, and the scar affixed to the bone group are shown in Table 3. BMP-2 expression levels were expressed in three different parts of the nonunion area, and were highest in the posted bone scar and lowest in the bone ends. The expression level in the posted bone scar was significantly different $(\mathrm{P}<0.05)$ to the canal content and bone ends groups. The sequence of BMP-2 expression levels in the three tissues was as follows: bone ends $<$ marrow cavity $<$ posted bone scar. DCN was expressed in three different parts of the nonunion area, and was highest in the bone ends. The expression level in the bone ends group was significantly different to the canal content and posted bone scar groups $(\mathrm{P}<0.05)$ (Table 3$)$.

\begin{tabular}{lccc}
\multicolumn{4}{c}{ Table 2. Bone morphogenetic protein 2 (BMP-2) and decorin $(\mathrm{DCN})$ staining in the three tissues (\%). } \\
\hline Group & Case No. & BMP-2 & DCN \\
\hline Bone ends & 11 & $0.30291 \pm 0.0958$ & $0.6165 \pm 0.2314$ \\
Canal content & 11 & $0.0856 \pm 0.0341$ & $0.0733 \pm 0.0171$ \\
Posted bone scar & 11 & $0.5886 \pm 0.0908$ & $0.2159 \pm 0.0974$ \\
F & & 51.370 & 18.789 \\
P & & 0.000 & 0.000 \\
\hline
\end{tabular}

The expression levels of BMP-2 and DCN were different in the bone ends, canal content, and posted bone scar groups. The comparisons of BMP-2 in the three groups by least significant difference (LSD) analysis, $\mathrm{P}<0.05$; comparisons of DCN expression by LSD between the fracture fragment group and the other two groups, $\mathrm{P}<0.05$; between the other two groups, $\mathrm{P}>0.05$.

Table 3. Expression levels of bone morphogenetic protein 2 (BMP-2) and decorin (DCN) in each group (means \pm SD).

\begin{tabular}{lccr}
\hline Group & Case No. & BMP-2 & DCN \\
\hline Bone ends & 11 & $0.3611 \pm 0.1646$ & $1.9740 \pm 0.9399$ \\
Canal content & 11 & $0.4670 \pm 0.1504$ & $0.9289 \pm 0.3189$ \\
Posted bone scar & 11 & $1.0583 \pm 0.2670$ & $0.6797 \pm 0.3113$ \\
F & & 27.995 & 10.461 \\
P & & 0.000 & 0.010 \\
\hline
\end{tabular}

The expression level of BMP-2 in the posted bone scar group was compared with the bone ends and canal content groups by least significant difference (LSD) analysis, $\mathrm{P}<0.05$; comparison between the bone ends and canal content groups, $\mathrm{P}>0.05$. The expression level of $\mathrm{DCN}$ in the bone ends group compared with the other two groups, $\mathrm{P}<0.05$; comparison between the canal content and posted bone scar groups, $\mathrm{P}>0.05$.

\section{DISCUSSION}

Fracture healing involves many complex local and systemic regulatory factors, such as growth and differentiation factors, hormones, cytokines, and extracellular matrix components (Joyce et al., 1990). BMP and its receptor play a very important role in the fracture healing process. When the fracture site is distant, or there is heavy loss of periosteum, affecting the function of osteoblast-related cells in the periosteum and bone marrow cavity, the fracture cannot be healed. Insufficient numbers of periosteal osteoblasts are transformed into osteoblasts and chondrocytes. At the same time, the osteogenic effect of endosteal osteoblasts and lobe cells in the bone marrow are also blocked. Various cells (such as fibroblasts, osteoblasts, and chondroblasts) often appear disordered and disorganized in various calluses. Fibroblasts in particular cannot undergo metaplasia into bone cells and bone tissue, and scars are formed at the fracture site. At the same time, the production of collagen by fibroblasts and bone matrix 
by osteoblasts is blocked, which affects osteogenesis. Additionally, osteoclasts are always active in the entire process of fracture healing. If phagocytosis is prevalent, the newly formed bone tissue is destroyed, and the bone callus cannot form. The fracture sites are linked up by the most vulnerable fibrous scars; they are very fragile, and cannot meet the mechanical requirements of body function.

Urist et al. (1984) successfully extracted BMP-2 and found that it can induce ectopic new bone formation. Many experiments have shown that BMP-2 shows highly efficient induction of osteogenic activity in a variety of animal models. BMP-2-induced bone formation produces bone within cartilage. The cartilage is formed first and then the bone is formed (Reddi and Anderson, 1976), which affects the recruitment and differentiation of undifferentiated mesenchymal cells and bone lineage (Riley et al., 1996). In the early stage of bone formation, BMP-2 can not only allow undifferentiated mesenchymal cells to gather at the bone formation center and differentiate into bone lineage cells, but can also make fibroblasts, myoblasts, and bone marrow stromal cells reverse-differentiate into osteoblasts. In late bone formation, BMP2 can also serve as an osteoclast differentiation factor by stimulating direct or indirect osteoclast differentiation with other support osteoclast differentiation factors (Long et al., 1995). Volek-Smith and Urist (1996) have induced osteogenesis using different doses of BMP-2, and they found that the bone formation content was proportional to the implant dose. Fleet et al. (1996) found that with increasing age of the animal, the BMP-2-induced osteogenic activity decreased, which may result from the reduced number of mesenchymal cells in older animals or reduced susceptibility of the mesenchymal cells to BMP-2. More recently, Lieberman et al. (2004) applied core decompression treatment in 17 cases of femoral head aseptic necrosis, and free fibula composite BMP-2 served as a pressure filler. The average follow-up was 4 years and 15 cases achieved a significant effect. Wang et al. (1990) found that a mouse mineral matrix composited with BMP-2 as a scaffold can induce bone formation. High doses of BMP-2 in the early stage can increase production of cartilage and bone formation, which confirms that BMP-2 plays a regulatory role in the bone induction process. A previous report has shown that a stroma-BMP-2 matrix implanted in mice induces bone formation (Wozney et al., 1990). Johnson and Urist (2000) utilized fresh frozen bone combined with BMP to treat 30 cases of femoral nonunion, at the same time correcting asymmetry until 6 months after recovery. This showed that the combination induced bone formation and remodeled the graft. According to the above theory and research, we conclude that BMP-2 has the ability to induce the healing of bone locally. However, for old tissue in the nonunion area, whether a local injection of BMP-2 would work, and what the specific effective site would be, remain unclear.

From the results of this experiment, the bone marrow cavity contains a large number of cells. However, most of these cells are incapable of bone turnover. This may be due to the local absence of BMP-2. Under the microscope, the tissues, which were not connected to the bone showed marrow fibrosis; in fibrous tissue that was connected to the bone and the fracture gap, we saw cartilage and fibrous ossification. The local environment was associated with the reduction of BMP-2 in the intramedullary and bone ends, which lacked an adequate blood supply; the reduction of BMP-2 caused interruptions in cell transformation and osteogenesis. Regardless of the detection level in mRNA or protein, the expression level of BMP-2 was highest in the posted bone scar. Organizational level inspection revealed that there was still some woven bone formation in a large amount of the fibrous tissue. At high magnification, there were more osteoblasts distributed around the neovascular tissue. Thus, in the nonunion area, there was still weak osteogenic capability, and these activities were mainly located in the 
bone paste scar near the bone ends. The endogenous BMP-2 mainly resides in the bone ends that affix to the bone scar. However, because small scars can be converted into a number of cells with bone cell activity, there was less formation of new bone. Scars are filled in with a great deal of fibrous material and cannot form real ossification centers, and therefore phagocytic cells are soon excluded. This is the main cause of termination of the fracture healing process. Therefore, according to the results of this experiment, endogenous BMP-2 exists in the fracture around the scar, and still has osteogenic activity. Application of exogenous BMP-2 to the treatment of nonunion by complementary injection should be applied to the bone ends and intramedullary tissue for maximum effect.

\section{Conflicts of interest}

The authors declare no conflict of interest.

\section{REFERENCES}

Fleet JC, Cashman K, Cox K and Rosen V (1996). The effects of aging on the bone inductive activity of recombinant human bone morphogenetic protein-2. Endocrinology 137: 4605-4610.

Groeneveld EH and Burger EH (2000). Bone morphogenetic proteins in human bone regeneration. Eur. J. Endocrinol. 142: 9-21.

Guerkov HH, Lohmann CH, Liu Y, Dean DD, et al. (2001). Pulsed electromagnetic fields increase growth factor release by nonunion cells. Clin. Orthop. Relat. Res. 384: 265-279.

Hietaniemi K, Paavolainen P and Penttinen R (1996). Connective tissue parameters in experimental nonunion. J. Orthop. Trauma 10: 114-118.

Hietaniemi K, Lehto MU and Paavolainen P (1998). Major fibrillar collagens and fibronectin in an experimental nonunion: an immunohistochemical study. Acta. Orthop. Scand. 69: 545-549.

Johnson EE and Urist MR (2000). Human bone morphogenetic protein allografting for reconstruction of femoral nonunion. Clin. Orthop. Relat. Res. 371: 61-74.

Johnson EE, Urist MR and Finerman GA (1988a). Bone morphogenetic protein augmentation grafting of resistant femoral nonunions. A preliminary report. Clin. Orthop. Relat. Res. 230: 257-265.

Johnson EE, Urist MR and Finerman GA (1988b). Repair of segmental defects of the tibia with cancellous bone grafts augmented with human bone morphogenetic protein. A preliminary report. Clin. Orthop. Relat. Res. 236: 249-257.

Johnson EE, Urist MR and Finerman GA (1990). Distal metaphysealtibial nonunion. Deformity and bone loss treated by open reduction, internal fixation, and human bone morphogenetic protein (hBMP). Clin. Orthop. Relat. Res. 250: 234-240.

Johnson EE, Urist MR and Finerman GA (1992). Resistant nonunions and partial or complete segmental defects of long bones.Treatment with implants of a composite of human bone morphogenetic protein (BMP) and autolyzed, antigenextracted, allogeneic (AAA) bone. Clin. Orthop. Relat. Res. 277: 229-237.

Joyce ME, Jingushi S and Bolander ME (1990). Transforming growth factor-beta in the regulation of fracture repair. Orthop. Clin. North Am. 21: 199-209.

Lieberman JR, Conduah A and Urist MR (2004). Treatment of osteonecrosis of the femoral head with core decompression and human bone morphogenetic protein. Clin. Orthop. Relat. Res. 429: 139-145.

Long MW, Robinson JA, Ashcraft EA and Mann KG (1995). Regulation of human bone marrow-derived osteoprogenitor cells by osteogenic growth factors. J. Clin. Invest. 95: 881-887.

Praemer A, Furner S and Rice DP (1992). Musculoskeletal injuries. In: Musculoskeletal Conditions in the United States. American Academy of Orthopaedic Surgeons, Park Ridge, 1992: 85-124.

Reddi AH (2001). Bone morphogenetic proteins: from basic science to clinical applications. J. Bone Joint Surg. Am. 83: S1-S6.

Reddi AH and Anderson WA (1976). Collagenous bone matrix-induced endochondral ossification hemopoiesis. J. Cell Biol. 69: 557-572.

Riley EH, Lane JM, Urist MR, Lyons KM, et al. (1996). Bone morphogenetic protein-2: biology and applications. Clin. Orthop. Relat. Res. 324: 39-46. 
Schmitz JP, Schwartz Z, Hollinger JO and Boyan BD (1990). Characterization of rat calvarial nonunion defects. Acta. Anat. 138: 185-192.

Urist MR, Huo YK, Brownell AG, Hohl WM, et al. (1984). Purification of bovine bone morphogenetic protein by hydroxyapatite chromatography. Proc. Natl. Acad. Sci. U. S. A. 81:371-375.

Volek-Smith H and Urist MR (1996). Recombinant human bone morphogenetic protein (rhBMP) induced heterotopic bone development in vivo and in vitro. Proc. Soc. Exp. Biol. Med. 211: 265-272.

Wang EA, Rosen V, D’Alessandro JS, Bauduy M, et al. (1990). Recombinant human bone morphogenetic protein induces bone formation. Proc. Natl. Acad. Sci. U. S. A. 87: 2220-2224.

Wozney JM, Rosen V, Byrne M, Celeste AJ, et al. (1990). Growth factors influencing bone development. J. Cell Sci. 13: $149-156$. 